\title{
Orthopädie und Geriatrie
}

Die Orthopädie ist definitionsgemäß u. a. mit der Behandlung von Patienten mit degenerativen Erkrankungen der Stuitz- und Bewegungsorgane betraut. Hierbei liegt das Interesse sowohl in der Diagnostik und letztendlich konservativen oder operativen Therapie, als auch in der klinischen und Grundlagenforschung.

Die Arthrosen der großen Gelenke wie Cox- und Gonarthrose ebenso wie die degenerativen Veränderungen der Wirbelsäule spielen hierbei zahlenmäßig die größte Rolle.

Allgemein ist festzustellen, daß diese orthopädischen Krankheitsbilder alle eine relativ strenge Alterskorrelation haben und somit bei Patienten höheren Lebensalters wesentlich häufiger zu finden sind.

Die durchschnittliche Lebenserwartung der Bevölkerung der Bundesrepublik Deutschland steigt. Sie hat sich innerhalb der letzten hundert Jahre verdoppelt, so daß auch die Anzahl der Menschen mit Erkrankungen der Stütz- und Bewegungsorgane deutlich zunimmt. Die volkswirtschaftlichen Kosten der degenerativen Gelenkerkrankungen sind enorm hoch. Die Gesamtkrankheitskosten der Arthrosen beliefen sich schon 1989 in Deutschland auf ca. DM 25 Mrd. Die degenerativen Gelenkerkrankungen stehen heute an der Spitze aller Invaliditätsursachen und sind so ein großes sozioökonomisches, volkswirtschaftliches, medizinisches und individuelles Problem

In unserer modernen Industriegesellschaft ist bei zunehmendem Lebensalter der Wunsch nach Selbständigkeit und Mobilität sehr hoch. Individuell für jeden Patienten ist die bedingt durch eine solche Erkrankung limitierte Lebensqualität durch Schmerzen und Einschränkung des Aktionsradius ein einschneidendes Erlebnis. Heutzutage wird ein solcher Zustand nicht zwangsläufig als Gott gegeben hingenommen, sondern versucht der drohenden Immobilität mit allen Mitteln entgegenzuwirken. Die aktuellen Behandlungsmöglichkeiten z. B. in der Endoprothetik aber auch in der Wirbelsäulenchirurgie z. B. bei Spinalkanalstenose tragen dieser Entwicklung Rechnung. Heute können Patienten in wesentlich höherem Lebensalter und mit höheren internistischen Risiken durch verbesserte operative aber auch anästhesiologische Therapieoptionen relativ sicher einem guten Behandlungsergebnis zugeführt werden. Auch hochbetagte Patienten können somit schmerzfrei und mobil erhalten werden und man kann ihnen somit ein großes Stück an Selbständigkeit bewahren.

Die mit den Aktivitäten der Geriatrie angesprochenen Patienten weisen in einem hohen Anteil gleichzeitig mehrere, dem Alterungsprozeß assoziierte Erkrankungen auf. Gerade hieraus ergibt sich die Forderung zu interdisziplinärer Zusammenarbeit. Eine weitere Konsequenz ist darüber hinaus, die Erstellung von Geriatriekonzepten und geriatrischen Zentren, eine Aktivität, die außer von Ärzten auch von Kostenträgern und politischen Entscheidungsträgern angedacht und aktiv bearbeitet wird. Das

Z. Orthop. 137 (1999) 467

(C) 1999 Georg Thieme Verlag Stuttgart · New York
Land Baden-Württemberg z. B. hat in seinem Geriatriekonzept geriatrischen Zentren die Aufgabe gestellt, geriatrische Patienten, an unterschiedlichen Krankheitsbildern leidend, zu versorgen, aber ihre Versorgung nicht nur zu sichern, sondern durch begleitende wissenschaftliche Untersuchungen zu verbessern. Daß hierbei der Aufbau interdisziplinärer Arbeitsgruppen unausweichlich ist, versteht sich von selbst und die interdisziplinäre Arbeitsgruppe hat im geriatrischen Konzept für die Versorgung der angesprochenen Patienten Prozeßqualität sicherzustellen.

Im vorliegenden Heft unserer wissenschaftlichen Zeitschrift haben wir versucht, wesentliche Aspekte der Geriatrie aus der Sicht von Orthopädie und Traumatologie zu beleuchten. Derzeitiges Wissen zur Therapie der Erkrankungen der Wirbelsäule, hier mit dem Schwerpunkt der Spinalkanalstenose, ist ebenso angesprochen wie degenerative Gelenkerkrankungen - hier insbesondere Cox- und Gonarthrose und in einem weiteren Schwerpunkt die Versorgung hüftgelenknaher Frakturen des geriatrischen $\mathrm{Pa}$ tienten. Besonderheiten der Versorgung des Hochbetagten sollen betont sein. Damit sollen Versorgungen angesprochen werden, die sowohl der auf der Akutstation tätige Arzt als auch der Geriater in der täglichen Arbeit beherrschen muß.

Epidemiologie und Grundlagenforschung müssen also neben der Beherrschung der klinischen Diagnostik und Therapie gewissermaßen die Zukunft vorbereiten. Eine Zukunft, die zwanghaft von demographischen Entwicklungen geprägt sein wird. Auch das Fach der Orthopädie sollte es keinesfalls versäumen, für sich und die Umgebung deutlich zu machen, welche Aufgabengebiete seit eh und je zentrale Arbeit des Orthopäden sind, Aufgabenbereiche, die auch durch die Schaffung eines geriatrischen Zentrums oder des Facharztes für Geriatrie nicht aus dem orthopädischen Bereich herausgelöst sind.

Fachlich hervorragende und informierte Arbeit des Orthopäden sind ebenso in der Zukunft gefragt wie die für unser Fach so typische Neigung und Erfordernis zu interdisziplinärer Arbeit. Letztendlich muß die interdisziplinäre Analyse der Verläufe auch deutlich machen, welche $\mathrm{Pa}$ tienten in der Zukunft zu ihrem eigenen Wohle und vielleicht sogar zum Wohle der Kostenträger in orthopädischen Kliniken und welche in geriatrischen Abteilungen zu versorgen sind. Der politisch aktive Entscheidungsträger für die Erstellung von Rahmenstrukturen wird dann den sachlichen Argumenten zu folgen haben.

Die DGOT als wissenschaftliche Gesellschaft unseres Faches muß Impulse geben, Maßstäbe setzen und sich an bereits getroffenen internationalen Maßstäben orientieren, sie aber andererseits für unser Land auch kritisch durch wissenschaftliche Daten hinterfragen. Klinische Forschung tut Not! Aus unserer Sicht können Forschungsschwerpunkte so $\mathrm{zu}$ ökonomischen Fragestellungen führen, aber auch im weiteren Sinne Arthroseforschung und die Bearbeitung der Epidemiologie degenerativer Wirbelsäulenerkrankungen sein. 